Stability estimates for the inverse boundary value problem by partial Cauchy data

This article has been downloaded from IOPscience. Please scroll down to see the full text article.

2006 Inverse Problems 221787

(http://iopscience.iop.org/0266-5611/22/5/015)

The Table of Contents and more related content is available

Download details:

IP Address: 140.112.113.225

The article was downloaded on 23/06/2009 at 13:21

Please note that terms and conditions apply. 


\title{
Stability estimates for the inverse boundary value problem by partial Cauchy data
}

\author{
Horst Heck $^{1}$ and Jenn-Nan Wang ${ }^{2}$ \\ ${ }^{1}$ Technische Universität Darmstadt, FB Mathematik, AG 4, Schlossgartenstr 7, \\ D-64289 Darmstadt, Germany \\ 2 Department of Mathematics, National Taiwan University, Taipei 106, Taiwan \\ E-mail: heck@mathematik.tu-darmstadt.de and jnwang@math.ntu.edu.tw
}

Received 5 January 2006, in final form 2 August 2006

Published 18 September 2006

Online at stacks.iop.org/IP/22/1787

\begin{abstract}
In this paper we study the inverse boundary value problem for the Schrödinger equation with a potential and the conductivity equation using partial Cauchy data. We derive stability estimates for these inverse problems.
\end{abstract}

\section{Introduction}

Let $n \geqslant 3$ and $\Omega \subset \mathbb{R}^{n}$ be an open bounded domain with $C^{\infty}$ boundary $\partial \Omega$ throughout the paper. Given $q(x) \in L^{\infty}(\Omega)$, we consider the boundary value problem

$$
\begin{cases}(\Delta-q) u=0 & \text { in } \Omega \\ u=f & \text { on } \partial \Omega,\end{cases}
$$

where $f \in H^{1 / 2}(\partial \Omega)$. Assume that 0 is not a Dirichlet eigenvalue of $\Delta-q$ on $\Omega$. Then (1.1) has a unique solution $u \in H^{1}(\Omega)$. The usual definition of the Dirichlet-to-Neumann map is given by

$$
\Lambda_{q} f=\left.\partial_{\nu} u\right|_{\partial \Omega}
$$

where $\partial_{\nu} u=\nabla u \cdot v$ and $v$ is the unit outer normal of $\partial \Omega$. The well-known inverse problem is to determine the potential $q$ from $\Lambda_{q}$. This problem was first proposed by Calderón [Ca80]. The uniqueness issue was settled by Sylvester and Uhlmann [SU87] and a reconstruction procedure was given by Nachman [Na88]. This inverse problem is known to be ill-posed. A log-type stability estimate was derived by Alessandrini [A188]. On the other hand, it was shown by Mandache [Ma01] that the log-type estimate is optimal.

All results mentioned above are concerned with the full data, i.e., measurements are made on the whole boundary. Recently, the inverse problem with partial data has attracted some attention. In [GU01] it is proved that one can determine the two-plane transform of the potential on a plane by measuring the Cauchy data of certain approximate solutions of the Schrödinger equation on a neighbourhood of the intersection of the plane with the boundary. 
A general uniqueness result with partial data was obtained by Bukhgeim and Uhlmann in [BU02] where the Neumann data were taken on a part of $\partial \Omega$ which is, roughly speaking, slightly larger than the half of the boundary. In [BU02], the Dirichlet data are imposed on the whole boundary. Bukhgeim and Uhlmann's result was recently improved to a more general case in [KSU05] where the Cauchy data can be taken on any part of the boundary. In this work, we derive a stability estimate for the inverse problem considered in [BU02]. Intuitively, we expect that the stability estimate with partial data is worse than that with full data. Indeed, our estimate turns out to be a log-log type.

To state the main result, we first introduce several notations. Picking a $\xi \in S^{n-1}$ and letting $\varepsilon>0$, we define

$$
\partial \Omega_{+, \varepsilon}=\{x \in \partial \Omega:\langle\xi, v(x)\rangle>\varepsilon\} \quad \partial \Omega_{-, \varepsilon}=\partial \Omega \backslash \overline{\partial \Omega_{+, \varepsilon}} .
$$

We also write $\partial \Omega_{+}=\partial \Omega_{+, 0}$ as well as $\partial \Omega_{-}=\partial \Omega_{-, 0}$. In order to study the stability estimate, we shall work in a more regular Sobolev space. Assume that $u \in H^{2}(\Omega)$ is the solution of (1.1) with $\left.u\right|_{\partial \Omega}=f \in H^{3 / 2}(\partial \Omega)$. We define the localized Dirichlet-to-Neumann map by

$$
\tilde{\Lambda}_{q}:\left.f \mapsto \partial_{\nu} u\right|_{\partial \Omega_{-, \varepsilon}} .
$$

So $\tilde{\Lambda}_{q}$ is a bounded operator from $H^{3 / 2}(\partial \Omega)$ to $\tilde{H}^{1 / 2}\left(\partial \Omega_{-, \varepsilon}\right)$ the restriction of $H^{1 / 2}(\partial \Omega)$ onto $\partial \Omega_{-, \varepsilon}$. The operator norm of $\tilde{\Lambda}_{q}$ is denoted by $\left\|\tilde{\Lambda}_{q}\right\|_{*}$.

Theorem 1.1. Let $N>0, s>\frac{n}{2}$ and $q_{j} \in H^{s}(\Omega)$ such that

$$
\left\|q_{j}\right\|_{H^{s}(\Omega)} \leqslant N
$$

for $j=1,2$, and 0 is not a Dirichlet eigenvalue of $\Delta-q_{j}, j=1,2$ then there exist constants $K, C>0$ and $\sigma_{1}, \sigma_{2} \in(0,1)$ such that

$$
\left\|q_{1}-q_{2}\right\|_{L^{\infty}(\Omega)} \leqslant C\left(\left\|\tilde{\Lambda}_{q_{1}}-\tilde{\Lambda}_{q_{2}}\right\|_{*}^{\sigma_{1}}+\left(\frac{1}{K} \log \left|\log \left\|\tilde{\Lambda}_{q_{1}}-\tilde{\Lambda}_{q_{2}}\right\|_{*}\right|\right)^{-\sigma_{2}}\right)
$$

where $K, C$ depend on $\Omega, N, \varepsilon, n, s$ and $\sigma_{1}, \sigma_{2}$ depend on $n, s$.

Theorem 1.1 can be generalized to the conductivity equation. Let $\gamma(x) \in H^{s}(\Omega)$ with $s>3+\frac{n}{2}$ be a strictly positive function on $\bar{\Omega}$. The equation for the electrical potential in the interior without sinks or sources is

$$
\begin{cases}\operatorname{div}(\gamma \nabla u)=0 & \text { in } \Omega \\ u=f & \text { on } \partial \Omega .\end{cases}
$$

The localized Dirichlet-to-Neumann map defined in this case is

$$
\tilde{\Lambda}_{\gamma}:\left.f \mapsto \gamma \partial_{\nu} u\right|_{\partial \Omega_{-, \varepsilon}}
$$

We can also see that $\tilde{\Lambda}_{\gamma}$ is a bounded map from $H^{3 / 2}(\partial \Omega)$ to $\tilde{H}^{1 / 2}\left(\partial \Omega_{-, \varepsilon}\right)$. Now theorem 1.1 implies that

Corollary 1.2. Assume that $\gamma_{j} \geqslant N^{-1}>0, s>\frac{n}{2}$, and

$$
\left\|\gamma_{j}\right\|_{H^{s+3}(\Omega)} \leqslant N
$$

for $j=1,2$, and

$$
\gamma_{1}=\gamma_{2} \quad \text { and } \quad \partial_{\nu} \gamma_{1}=\partial_{\nu} \gamma_{2} \text { on } \overline{\partial \Omega_{+, \varepsilon}} .
$$

Then there exist constants $K, C>0$ and $\sigma, \tilde{\sigma}_{1}, \tilde{\sigma}_{2} \in(0,1)$ such that

$$
\left\|\gamma_{1}-\gamma_{2}\right\|_{L^{\infty}(\Omega)} \leqslant C\left(\left\|\tilde{\Lambda}_{\gamma_{1}}-\tilde{\Lambda}_{\gamma_{2}}\right\|_{*}^{\tilde{\sigma}_{1}}+\left(\frac{1}{K} \log \left(\frac{1}{\sigma}\left|\log \left\|\tilde{\Lambda}_{\gamma_{1}}-\tilde{\Lambda}_{\gamma_{2}}\right\|_{*}\right|\right)\right)^{-\tilde{\sigma}_{2}}\right)
$$

where $K, C$ depend on $\Omega, N, \varepsilon, n, s$ and $\sigma, \tilde{\sigma}_{1}, \tilde{\sigma}_{2}$ depend on $n, s$. 
The main strategy in deriving the estimate (1.2) is to adapt Bukhgeim and Uhlmann's arguments to the case $\tilde{\Lambda}_{1} \neq \tilde{\Lambda}_{2}$. At the end, we will get an estimate of the Fourier transform of $q:=q_{1}-q_{2}$ on some subset of $\mathbb{R}^{n}$. Since we can treat $q$ as a compactly supported function, its Fourier transform is real analytic. We then modify Vessella's stability estimate for analytic continuation [Ve99] to our case here. Note that we do not claim any optimality of our estimate. Also, we want to remark that even allowing $\varepsilon$ to be sufficiently large, our stability estimate does not include the well-known stability result of Alessandrini [A188] for the case of full Cauchy data. This is due to the type of stability estimate for analytic continuation used in the method. For the full data problem, Alessandrini [A188] used a different stability estimate for analytic continuation and obtained a log type estimate. However, we are not able to use this estimate in the partial data problem. In fact, for the full data problem the analytic continuation procedure is not needed [A189].

\section{Preliminaries}

In this section we collect some results from [BU02] and [Ve99] which are needed in the proof of theorem 1.1. The first one is the Carleman estimate for the operator $\Delta-q$.

Proposition 2.1 [BU02 corollary 2.3]. For any $q \in L^{\infty}(\Omega)$ there exist $\tilde{\tau}_{0}, C>0$ such that for all $u \in H^{2}(\Omega) \cap H_{0}^{1}(\Omega)$ and $\tau \geqslant \tilde{\tau}_{0}$, then

$$
\begin{aligned}
& C \tau^{2} \int_{\Omega}\left|\mathrm{e}^{-\tau\langle x, \xi\rangle} u\right|^{2} \mathrm{~d} x+\tau \int_{\partial \Omega_{+}}\langle\xi, v\rangle\left|\mathrm{e}^{-\tau\langle x, \xi\rangle} \partial_{\nu} u\right|^{2} \mathrm{~d} S \\
& \quad \leqslant \int_{\Omega}\left|\mathrm{e}^{-\tau\langle x, \xi\rangle}(\Delta-q) u\right|^{2} \mathrm{~d} x-\tau \int_{\partial \Omega_{-}}\langle\xi, v\rangle\left|\mathrm{e}^{-\tau\langle x, \xi\rangle} \partial_{\nu} u\right|^{2} \mathrm{~d} S,
\end{aligned}
$$

where $C=\tilde{\tau}_{0} / 2$ and $\tilde{\tau}_{0}$ is chosen such that

$$
\tilde{\tau}_{0}>\frac{1}{2}\|q\|_{L^{\infty}(\Omega)} \sqrt{\operatorname{diam}(\Omega)} .
$$

In our proof, we shall use complex geometrical optics solutions for the Schrödinger operator $\Delta-q$ which were first introduced by Sylvester and Uhlmann [SU87]. More precisely, for $\rho \in \mathbb{C}^{n}$ with $\langle\rho, \rho\rangle=0$ and $\rho=\tau(\xi+\mathrm{i} \eta), \xi, \eta \in S^{n-1}$, we can find $u_{\rho}=\mathrm{e}^{\langle x, \rho\rangle}\left(1+\psi_{q}(x, \rho)\right)$ such that

$$
(\Delta-q) u_{\rho}=0 \quad \text { in } \Omega
$$

and for some $C=C\left(\Omega, t,\|q\|_{L^{\infty}(\Omega)}\right)>0$

$$
\left\|\psi_{q}(\cdot, \rho)\right\|_{H^{t}(\Omega)} \leqslant \frac{C}{\tau^{1-t}}
$$

where $0 \leqslant t \leqslant 2$ and $\tau \geqslant \hat{\tau}_{0}=\hat{\tau}_{0}\left(\Omega,\|q\|_{L^{\infty}(\Omega)}\right)$. We now denote $\tau_{0}=\max \left\{\tilde{\tau}_{0}, \hat{\tau}_{0}\right\}$. Thus, $\tau_{0}$ depends on $\Omega$ and $\|q\|_{L^{\infty}(\Omega)}$.

The following result on continuous dependence in the analytic continuation problem will be very important to prove our main result. It is due to Vessella.

Proposition 2.2 [Ve99, theorem 1]. Let $r_{0}, d_{0}>0$. Let $D \subset \mathbb{R}^{n}$ be an open, bounded and connected set such that $\{x \in D: d(x, \partial D)>r\}$ is connected for any $r \in\left[0, r_{0}\right]$. Further let $E \subset D$ be an open set such that $d(E, \partial D) \geqslant d_{0}$. If $f$ is an analytic function with

$$
\left\|\partial^{\alpha} f\right\|_{L^{\infty}(D)} \leqslant \frac{M \alpha !}{\zeta^{|\alpha|}}, \quad \text { for all } \quad \alpha \in \mathbb{N}^{n}
$$


for some $M, \zeta>0$ then

$$
|f(x)| \leqslant(2 M)^{1-\gamma_{1}(|E| /|D|)}\left(\|f\|_{L^{\infty}(E)}\right)^{\gamma_{1}(|E| /|D|)},
$$

where $\gamma_{1} \in(0,1)$ depends on $d_{0}$, diam $D, n, r_{0}, \zeta$ and $d(x, \partial D)$.

We will use this result in the special case $D=B(0,2)$ and $E$ is an open subset of $B(0,1)$. Most importantly, we have to find a $\gamma_{1}$ such that (2.3) is valid for all $x \in B(0,1)$. To this end, we need to express $\gamma_{1}$ explicitly from [Ve99]. In [Ve99] (p 702), one has

$$
\gamma_{1}=\mu \exp \left[\left(\frac{2(12 \sqrt{n})^{n}|D|}{\omega_{n} r_{2}^{n}}+3\right) \ln \beta_{1}\right],
$$

where $\omega_{n}$ is the measure of the $n$-dimensional unit ball, $\mu=\mu\left(d_{0}\right.$, diam $\left.D, n, \zeta\right)$, and

$$
\beta_{1}=\frac{\sinh \left(\frac{2 \pi A}{n \zeta}\right)}{2\left(\sinh \frac{5 \pi A}{n \zeta}\right)\left(\cosh \frac{\pi n \zeta}{4 A}\right)} \quad \text { with } \quad A=\frac{r_{2}}{12 \sqrt{n}} .
$$

Here, $r_{2}=\min \left\{d(x, \partial D), r_{1}\right\}$ and $r_{1}=\min \left\{r_{0}, d_{0} / 2\right\}$.

\section{Stability estimate for the potential}

In this section we prove the main result of this paper theorem 1.1. We are going to use the complex geometrical optics solutions and the Carleman estimate to get control of the Fourier transform of the difference of two potentials on a subset of $\mathbb{R}^{n}$. Then the estimation for the analytic continuation gives us control on all of $\mathbb{R}^{n}$ and this makes possible our stability estimates.

As before we let $\xi \in S^{n-1}$. Fix $k \in \mathbb{R}^{n}$ satisfying $\langle\xi, k\rangle=0$. We now set $\rho_{1}=$ $-\tau \xi-\mathrm{i}\left(\frac{k-\eta}{2}\right)$ and $\rho_{2}=\tau \xi-\mathrm{i}\left(\frac{k+\eta}{2}\right)$ such that $\langle\xi, \eta\rangle=\langle k, \eta\rangle=0$ and $|k+\eta|^{2}=4 \tau^{2}$. Then we can see that $\left\langle\rho_{j}, \rho_{j}\right\rangle=0$ for $j=1,2$. Let $u_{2}=\mathrm{e}^{\left\langle x, \rho_{2}\right\rangle}\left(1+\psi_{q_{2}}\right)$ be a solution of $\left(\Delta-q_{2}\right) u_{2}=0$ and let $\bar{v}=\mathrm{e}^{\left\langle x, \rho_{1}\right\rangle}\left(1+\psi_{q_{1}}\right)$ be a solution of $\left(\Delta-q_{1}\right) \bar{v}=0$. We denote by $u_{1}$ the solution of

$$
\left(\Delta-q_{1}\right) u_{1}=0 \quad \text { in } \Omega \quad \text { and }\left.\quad u_{1}\right|_{\partial \Omega}=\left.u_{2}\right|_{\partial \Omega}=: f .
$$

Defining $u=u_{1}-u_{2}$ and $q=q_{1}-q_{2}$ we get that

$$
\left(\Delta-q_{1}\right) u=q u_{2} \quad \text { in } \Omega \quad \text { and }\left.\quad u\right|_{\partial \Omega}=0 .
$$

Since $q u_{2} \in L^{2}(\Omega)$ it is clear that $u \in H^{2}(\Omega)$. Multiplying this equation with $\bar{v}$ and integrating over $\Omega$ we obtain by using the Green's formula

$$
\int_{\Omega} q u_{2} \bar{v} \mathrm{~d} x=\int_{\partial \Omega_{+, \varepsilon}}\left(\partial_{\nu} u\right) \bar{v} \mathrm{~d} S+\int_{\partial \Omega_{-, \varepsilon}}\left(\partial_{\nu} u\right) \bar{v} \mathrm{~d} S,
$$

where $\mathrm{d} S$ denotes the surface measure on $\partial \Omega$. We further have that $\left(\Lambda_{q_{1}}-\Lambda_{q_{2}}\right) f=\left.\partial_{\nu} u\right|_{\partial \Omega}$.

Using the Carleman estimate (2.1) for $q_{1}$ and the trace theorem as well as inequality (2.2) for $\psi_{q_{1}}$, we estimate the first term on the right-hand side of (3.1) by

$$
\begin{aligned}
\left|\int_{\partial \Omega_{+, \varepsilon}}\left(\partial_{\nu} u\right) \bar{v} \mathrm{~d} S\right| \leqslant & \left\|1+\psi_{q_{1}}\right\|_{L^{2}\left(\partial \Omega_{+, \varepsilon}\right)}\left\|\mathrm{e}^{-\tau\langle x, \xi\rangle} \partial_{\nu} u\right\|_{L^{2}\left(\partial \Omega_{+, \varepsilon}\right)} \\
\leqslant & \left(\left|\partial \Omega_{+, \varepsilon}\right|^{\frac{1}{2}}+\left\|\psi_{q_{1}}\right\|_{H^{1}(\Omega)}\right)\left((\tau \varepsilon)^{-1} \int_{\Omega}\left|\mathrm{e}^{-\tau\langle x, \xi\rangle} q u_{2}\right|^{2} \mathrm{~d} x\right. \\
& \left.-\inf _{x \in \partial \Omega_{-, \varepsilon}}\langle\xi, v(x)\rangle \varepsilon^{-1} \int_{\partial \Omega_{-, \varepsilon}}\left|\mathrm{e}^{-\tau\langle x, \xi\rangle} \partial_{\nu} u\right|^{2} \mathrm{~d} S\right)^{\frac{1}{2}} \\
\leqslant & C\left(\tau^{-1} \int_{\Omega}\left|\mathrm{e}^{-\tau\langle x, \xi\rangle} q u_{2}\right|^{2} \mathrm{~d} x+\int_{\partial \Omega_{-, \varepsilon}}\left|\mathrm{e}^{-\tau\langle x, \xi\rangle} \partial_{\nu} u\right|^{2} \mathrm{~d} S\right)^{\frac{1}{2}}
\end{aligned}
$$


for $\tau \geqslant \tau_{0}$, where $C=C(\Omega, \varepsilon, N)$ and $\tau_{0}=\tau_{0}(\Omega, N)$. Setting in the definition of $v$ into the second term on the right-hand side of (3.1) gives

$\left|\int_{\partial \Omega_{-, \varepsilon}}\left(\partial_{\nu} u\right) \bar{v} \mathrm{~d} S\right| \leqslant \int_{\partial \Omega_{-, \varepsilon}}\left|\mathrm{e}^{-\tau\langle x, \xi\rangle}\left(\partial_{\nu} u\right)\left(1+\psi_{q_{1}}\right)\right| \mathrm{d} S \leqslant C\left(\int_{\partial \Omega_{-, \varepsilon}}\left|\mathrm{e}^{-\tau\langle x, \xi\rangle} \partial_{\nu} u\right|^{2} \mathrm{~d} S\right)^{\frac{1}{2}}$.

Also, here we note $C=C(\Omega, \varepsilon, N)$. In other words, we have that

$$
\begin{aligned}
& \mid \int_{\partial \Omega_{+, \varepsilon}}\left(\partial_{\nu} u\right) \bar{v} \mathrm{~d} S+\int_{\partial \Omega_{-, \varepsilon}}\left(\partial_{\nu} u\right) \bar{v} \mathrm{~d} S \mid \\
& \leqslant C\left(\tau^{-1} \int_{\Omega}\left|\mathrm{e}^{-\tau\langle x, \xi\rangle} q u_{2}\right|^{2} \mathrm{~d} x+\int_{\partial \Omega_{-, \varepsilon}}\left|\mathrm{e}^{-\tau\langle x, \xi\rangle} \partial_{\nu} u\right|^{2} \mathrm{~d} S\right)^{\frac{1}{2}} .
\end{aligned}
$$

Since

$$
\int_{\Omega} q u_{2} \bar{v} \mathrm{~d} x=\int_{\Omega} \mathrm{e}^{-\mathrm{i}\langle k, x\rangle} q\left(1+\psi_{q_{1}}+\psi_{q_{2}}+\psi_{q_{1}} \psi_{q_{2}}\right) \mathrm{d} x
$$

and

$$
\int_{\partial \Omega_{-, \varepsilon}}\left|\partial_{\nu} u\right|^{2} \mathrm{~d} S=\left\|\left(\tilde{\Lambda}_{q_{1}}-\tilde{\Lambda}_{q_{2}}\right) f\right\|_{L^{2}\left(\partial \Omega_{-, \varepsilon}\right)}^{2} \leqslant\left\|\tilde{\Lambda}_{q_{1}}-\tilde{\Lambda}_{q_{2}}\right\|_{*}^{2}\left\|u_{2}\right\|_{H^{2}(\Omega)}^{2},
$$

we finally get from (2.2), (3.1), (3.2), and the a priori assumption of $q_{1}, q_{2}$ that

$$
\begin{aligned}
\left|\int_{\Omega} \mathrm{e}^{-\mathrm{i}\langle k, x\rangle} q \mathrm{~d} x\right|^{2} \leqslant & C\left|\int_{\Omega} \mathrm{e}^{-\mathrm{i}\langle k, x\rangle} q\left(\psi_{q_{1}}+\psi_{q_{2}}+\psi_{q_{1}} \psi_{q_{2}}\right) \mathrm{d} x\right|^{2} \\
& +C\left(\tau^{-1} \int_{\Omega}\left|q\left(1+\psi_{q_{2}}\right)\right|^{2} \mathrm{~d} x+\mathrm{e}^{c \tau} \int_{\partial \Omega_{-, \varepsilon}}\left|\partial_{\nu} u\right|^{2} \mathrm{~d} S\right) \\
\leqslant & C\left(\tau^{-1}+\mathrm{e}^{c \tau}\left\|\tilde{\Lambda}_{q_{1}}-\tilde{\Lambda}_{q_{2}}\right\|_{*}^{2}\left\|u_{2}\right\|_{H^{2}(\Omega)}^{2}\right) \\
\leqslant & C\left(\mathrm{e}^{c(|k|+\tau)}\left\|\tilde{\Lambda}_{q_{1}}-\tilde{\Lambda}_{q_{2}}\right\|_{*}^{2}+\tau^{-1}\right)
\end{aligned}
$$

where both $C$ and $c$ depend on $\Omega, \varepsilon$, and $N$. Varying $\xi$ in a small conic neighbourhood $U \subset S^{n-1}$ we get the above estimate uniformly for all $k \in E=\left\{x \in \mathbb{R}^{n}: x\right.$ orthogonal to some $\left.\tilde{\xi} \in U(\xi) \subset S^{n-1}\right\}$. Note that for $t>0$ the equality $t E=\{t x: x \in E\}=E$ holds.

Next we set for fixed $R>0$, which will be made precise later, and $k \in \mathbb{R}^{n} f(k)=$ $(\mathcal{F} \tilde{q})(R k)$. Here $\mathcal{F}$ is the Fourier transform and $\tilde{q}$ denotes the trivial extension of $q$ to $\mathbb{R}^{n}$. Then it is clear that $f$ is analytic and

$\left|D^{\alpha} f(k)\right| \leqslant\|q\|_{L^{1}(\Omega)} \frac{R^{|\alpha|}}{\left(\operatorname{diam}(\Omega)^{-1}\right)^{|\alpha|}} \leqslant C \frac{R^{|\alpha|}}{\alpha !\left(\operatorname{diam}(\Omega)^{-1}\right)^{|\alpha|}} \alpha ! \leqslant C \frac{\mathrm{e}^{n R}}{\left(\operatorname{diam}(\Omega)^{-1}\right)^{|\alpha|}} \alpha !$

for any $\alpha \in \mathbb{N}^{n}$ with $C=C(N)$, where we have used the estimate

$$
\frac{R^{m}}{m !} \leqslant \mathrm{e}^{R}
$$

uniformly for all $m \in \mathbb{N} \cup\{0\}$ and $R>0$. In order to apply proposition 2.2 to $f$ on the domain $D=B(0,2)$, and the set $\tilde{E}=E \cap B(0,1)$ with $M=C \mathrm{e}^{n R}$ and $\zeta=\operatorname{diam}(\Omega)^{-1}$, we first calculate $r_{1}=\min \left\{r_{0}, d_{0} / 2\right\}=\min \{2,1 / 2\}=1 / 2, r_{2}=\min \left\{d(x, \partial D), r_{1}\right\}=$ $\min \{d(x, \partial D), 1 / 2\}=1 / 2$ for all $x \in B(0,1)$. To compute $|\tilde{E}|$, it suffices to choose $\xi=(1,0, \ldots, 0)$ by suitably rotating $\tilde{E}$. Hence, there exists a positive number $\varrho=\varrho(\varepsilon)$ with $\varrho<\pi / 2$ and

$$
\begin{aligned}
|\tilde{E}| & =\int_{0}^{1} \int_{\pi / 2-\varrho}^{\pi / 2+\varrho} \int_{0}^{\pi} \cdots \int_{0}^{\pi} \int_{0}^{2 \pi} r^{n-1} \sin ^{n-2} \theta_{1} \sin ^{n-3} \theta_{2} \cdots \sin \theta_{n-2} \mathrm{~d} r \mathrm{~d} \theta_{1} \mathrm{~d} \theta_{2} \cdots \mathrm{d} \theta_{n-2} \mathrm{~d} \theta_{n-1} \\
& =: d(n, \varepsilon)>0 .
\end{aligned}
$$


Subsequently, we have

$$
\frac{|\tilde{E}|}{|D|}=\frac{d(n, \varepsilon)}{2^{n} \omega_{n}}
$$

which is a positive constant (less than 1) depending on $n$ and $\varepsilon$. Therefore, with

$$
\theta=\gamma_{1} \frac{|\tilde{E}|}{|D|}=\mu \exp \left[\left(\frac{2(12 \sqrt{n})^{n}|D|}{\omega_{n} r_{2}^{n}}+3\right) \ln \beta_{1}\right] \frac{|\tilde{E}|}{|D|} \in(0,1)
$$

depending on $\varepsilon, n$, and $\operatorname{diam}(\Omega)$, we get from proposition 2.2 that

$|\mathcal{F} \tilde{q}(k)|=|f(k / R)| \leqslant C \mathrm{e}^{n R(1-\theta)}\|f\|_{L^{\infty}(\tilde{E})}^{\theta} \leqslant C \mathrm{e}^{n R(1-\theta)}\left(\|\mathcal{F} \tilde{q}(k)\|_{L^{\infty}(E)}\right)^{\theta}$

holds for all $k \in B(0, R)$.

Using (3.4) together with (3.3) we get

$$
\begin{aligned}
\|\tilde{q}\|_{H^{-1}}^{\frac{2}{\theta}} & =\left(\int_{|k| \leqslant R}|\mathcal{F} \tilde{q}|^{2}\left(1+|k|^{2}\right)^{-1} \mathrm{~d} k+\int_{|k|>R}|\mathcal{F} \tilde{q}|^{2}\left(1+|k|^{2}\right)^{-1} \mathrm{~d} k\right)^{\frac{1}{\theta}} \\
& \leqslant C\left(R^{n}\|\mathcal{F} \tilde{q}\|_{L^{\infty}(B(0, R))}^{2}+\frac{1}{R^{2}}\|q\|_{L^{2}(\Omega)}^{2}\right)^{\frac{1}{\theta}} \\
& \leqslant C\left(R^{\frac{n}{\theta}} \mathrm{e}^{2 n R \frac{1-\theta}{\theta}} \mathrm{e}^{c(R+\tau)}\left\|\tilde{\Lambda}_{q_{1}}-\tilde{\Lambda}_{q_{2}}\right\|_{*}^{2}+R^{\frac{n}{\theta}} \mathrm{e}^{2 n R \frac{1-\theta}{\theta}} \tau^{-1}+R^{-\frac{2}{\theta}}\right) .
\end{aligned}
$$

Choosing

$$
\tau=R^{\frac{n+2}{\theta}} \mathrm{e}^{2 n R \frac{1-\theta}{\theta}}
$$

such that $R^{\frac{n}{\theta}} \mathrm{e}^{2 n R \frac{1-\theta}{\theta}} \tau^{-1}=R^{-\frac{2}{\theta}}$ we proceed

$\|\tilde{q}\|_{H^{-1}\left(\mathbb{R}^{n}\right)}^{\frac{2}{\theta}} \leqslant C\left(R^{\frac{n}{\theta}} \exp \left(2 n R \frac{1-\theta}{\theta}+c\left(R+R^{\frac{n+2}{\theta}} \mathrm{e}^{2 n R \frac{1-\theta}{\theta}}\right)\right)\left\|\tilde{\Lambda}_{q_{1}}-\tilde{\Lambda}_{q_{2}}\right\|_{*}^{2}+R^{-\frac{2}{\theta}}\right)$.

It is not difficult to check that

$$
\begin{aligned}
& R^{\frac{n}{\theta}} \exp \left(2 n R \frac{1-\theta}{\theta}+c\left(R+R^{\frac{n+2}{\theta}} \mathrm{e}^{2 n R \frac{1-\theta}{\theta}}\right)\right) \\
& \leqslant \exp \left(\mathrm{e}^{\left[\frac{n}{\theta}+2 n \frac{1-\theta}{\theta}+c+\frac{n+2}{\theta}+2 n \frac{1-\theta}{\theta}\right] R}\right) \quad \text { for all } \quad R>0 .
\end{aligned}
$$

Setting $K=\frac{n}{\theta}+2 n \frac{1-\theta}{\theta}+c+\frac{n+2}{\theta}+2 n \frac{1-\theta}{\theta}$, (3.6) and (3.7) lead to

$$
\|\tilde{q}\|_{H^{-1}\left(\mathbb{R}^{n}\right)}^{\frac{2}{\theta}} \leqslant C\left(\mathrm{e}^{\mathrm{e}^{K R}}\left\|\tilde{\Lambda}_{q_{1}}-\tilde{\Lambda}_{q_{2}}\right\|_{*}^{2}+R^{-\frac{2}{\theta}}\right) .
$$

The arguments above are valid if $\tau \geqslant \tau_{0}$. By (3.5) we need to take $R$ sufficiently large. So there exists a $\delta \ll 1$ such that if $\left\|\tilde{\Lambda}_{q_{1}}-\tilde{\Lambda}_{q_{2}}\right\|_{*}<\delta$ and $R=\frac{1}{K} \log \left|\log \left\|\tilde{\Lambda}_{q_{1}}-\tilde{\Lambda}_{q_{2}}\right\|_{*}\right|$ we have $\tau \geqslant \tau_{0}$. To be more precise, we get from (3.5) that if

$$
\tau_{0} \leqslant \tau=R^{\frac{n+2}{\theta}} \mathrm{e}^{2 n R \frac{1-\theta}{\theta}} \leqslant \mathrm{e}^{R \frac{n+2}{\theta}} \mathrm{e}^{2 n R \frac{1-\theta}{\theta}}
$$

then

$$
R \geqslant \frac{\theta}{3 n+2-2 n \theta} \log \tau_{0}:=R_{0}
$$

In other words, we will need to take $\delta \leqslant \delta_{0}<1$ with

$$
\delta_{0} \leqslant \mathrm{e}^{-\mathrm{e}^{K R_{0}}} \text {. }
$$

So $\delta_{0}$ depends on $n, \varepsilon, \Omega$, and $N$. Thus it follows from (3.8) that

$$
\|q\|_{H^{-1}(\Omega)} \leqslant\|\tilde{q}\|_{H^{-1}\left(\mathbb{R}^{n}\right)} \leqslant C\left(\left\|\tilde{\Lambda}_{q_{1}}-\tilde{\Lambda}_{q_{2}}\right\|_{*}+\left(\frac{1}{K} \log \left|\log \left\|\tilde{\Lambda}_{q_{1}}-\tilde{\Lambda}_{q_{2}}\right\|_{*}\right|\right)^{-\frac{2}{\theta}}\right)^{\frac{\theta}{2}}
$$


when $\left\|\tilde{\Lambda}_{q_{1}}-\tilde{\Lambda}_{q_{2}}\right\|_{*}<\delta$. Now if $\left\|\tilde{\Lambda}_{q_{1}}-\tilde{\Lambda}_{q_{2}}\right\|_{*} \geqslant \delta$ then we have

$$
\|q\|_{H^{-1}(\Omega)} \leqslant C\|q\|_{L^{\infty}(\Omega)} \leqslant \frac{2 C N}{\delta^{\theta / 2}} \delta^{\theta / 2} \leqslant \frac{2 C N}{\delta^{\theta / 2}}\left\|\tilde{\Lambda}_{q_{1}}-\tilde{\Lambda}_{q_{2}}\right\|_{*}^{\frac{\theta}{2}} .
$$

Therefore, (3.9) also holds.

The estimate (1.2) is now an easy consequence of the interpolation theorem. Precisely, let $\epsilon>0$ such that $s=\frac{n}{2}+2 \epsilon$. Using that $\left[H^{t_{0}}(\Omega), H^{t_{1}}(\Omega)\right]_{\beta}=H^{t}(\Omega)$ with $t=$ $(1-\beta) t_{0}+\beta t_{1}$ (see e.g. [Tr95 theorem 1 in 4.3.1]) and the Sobolev embedding theorem, we get $\left\|q_{1}-q_{2}\right\|_{L^{\infty}} \leqslant C\left\|q_{1}-q_{2}\right\|_{H^{\frac{n}{2}+\epsilon}} \leqslant C\left\|q_{1}-q_{2}\right\|_{H^{t_{0}}}^{(1-\beta)}\left\|q_{1}-q_{2}\right\|_{H^{t_{1}}}^{\beta}$. Setting $t_{0}=-1$ and $t_{1}=s$ we end up with

$$
\left\|q_{1}-q_{2}\right\|_{L^{\infty}(\Omega)} \leqslant C\left\|q_{1}-q_{2}\right\|_{H^{-1}(\Omega)}^{\frac{s+1-\epsilon}{s+1}}
$$

which yields the desired estimate (1.2) with $\sigma_{1}=\frac{\theta}{2} \frac{s+1-\epsilon}{s+1}$ and $\sigma_{2}=\frac{s+1-\epsilon}{s+1}$.

\section{Stability estimate for the conductivity}

We will prove corollary 1.2 in this section. We recall the following well-known relation: let $q=\frac{\Delta \sqrt{\gamma}}{\sqrt{\gamma}}$ then

$$
\Lambda_{q}(f)=\left.\gamma^{-1 / 2}\right|_{\partial \Omega} \Lambda_{\gamma}\left(\left.\gamma^{-1 / 2}\right|_{\partial \Omega} f\right)+\left.\frac{1}{2}\left(\gamma^{-1} \partial_{\nu} \gamma\right)\right|_{\partial \Omega} f
$$

and similarly for the localized Dirichlet-to-Neumann map

$$
\tilde{\Lambda}_{q}(f)=\left.\gamma^{-1 / 2}\right|_{\partial \Omega_{-, \varepsilon}} \tilde{\Lambda}_{\gamma}\left(\left.\gamma^{-1 / 2}\right|_{\partial \Omega} f\right)+\left.\left.\frac{1}{2}\left(\gamma^{-1} \partial_{\nu} \gamma\right)\right|_{\partial \Omega_{-, \varepsilon}} f\right|_{\partial \Omega_{-, \varepsilon}} .
$$

Therefore, in order to apply theorem 1.1, we need to estimate the conductivity $\gamma$ on the boundary by $\tilde{\Lambda}_{\gamma}$. The first boundary determination result was given by Kohn and Vogelius [KV84] and a stability estimate on the boundary can be obtained by their method [A188]. Another boundary stability estimate was derived in [SU88]. We remark that both methods in [KV84] and [SU88] are local. So the same estimates should hold for the localized Dirichlet-to-Neumann map. In view of the a priori assumption of $\gamma_{j}$, we have at least $\gamma_{j}, \partial_{\nu} \gamma_{j} \in C(\partial \Omega), j=1,2$. Moreover, from (1.4) we see that

$$
\left\|\gamma_{1}-\gamma_{2}\right\|_{L^{\infty}(\partial \Omega)}=\left|\left(\gamma_{1}-\gamma_{2}\right)\left(p_{0}\right)\right|
$$

and

$$
\left\|\partial_{\nu} \gamma_{1}-\partial_{\nu} \gamma_{2}\right\|_{L^{\infty}(\partial \Omega)}=\left|\left(\partial_{\nu} \gamma_{1}-\partial_{\nu} \gamma_{2}\right)\left(p_{1}\right)\right|
$$

for some $p_{0}, p_{1} \in \partial \Omega_{-, \varepsilon}$. Therefore, the same arguments in [Al88] give

$$
\left\|\gamma_{1}-\gamma_{2}\right\|_{L^{\infty}(\partial \Omega)} \leqslant C\left\|\tilde{\Lambda}_{\gamma_{1}}-\tilde{\Lambda}_{\gamma_{2}}\right\|_{\frac{1}{2},-\frac{1}{2}}
$$

and

$$
\left\|\partial_{\nu} \gamma_{1}-\partial_{\nu} \gamma_{2}\right\|_{L^{\infty}(\partial \Omega)} \leqslant C\left(\left\|\tilde{\Lambda}_{\gamma_{1}}-\tilde{\Lambda}_{\gamma_{2}}\right\|_{\frac{1}{2},-\frac{1}{2}}^{\tilde{\theta}}+\left\|\tilde{\Lambda}_{\gamma_{1}}-\tilde{\Lambda}_{\gamma_{2}}\right\|_{\frac{1}{2},-\frac{1}{2}}\right)
$$

for some $0<\tilde{\theta}<1$ depending only on $n$. Using the interpolation of operators between $H^{\frac{3}{2}}(\partial \Omega) \rightarrow H^{\frac{1}{2}}(\partial \Omega)$ and $H^{-\frac{1}{2}}(\partial \Omega) \rightarrow H^{-\frac{3}{2}}(\partial \Omega)$ and the a priori assumption of $\gamma_{j}$, we get that

$$
\left\|\gamma_{1}-\gamma_{2}\right\|_{L^{\infty}(\partial \Omega)} \leqslant C\left\|\tilde{\Lambda}_{\gamma}-\tilde{\Lambda}_{\gamma_{2}}\right\|_{*}^{\frac{1}{2}}
$$


and

$$
\left\|\partial_{\nu} \gamma_{1}-\partial_{\nu} \gamma_{2}\right\|_{L^{\infty}(\partial \Omega)} \leqslant C\left(\left\|\tilde{\Lambda}_{\gamma}-\tilde{\Lambda}_{\gamma_{2}}\right\|_{*}^{\theta}+\left\|\tilde{\Lambda}_{\gamma_{1}}-\tilde{\Lambda}_{\gamma_{2}}\right\|_{*}^{\frac{1}{2}}\right)
$$

with $\theta=\frac{1}{2} \tilde{\theta}$. Note that the Dirichlet-to-Neumann map can be extended to a bounded operator from $H^{-\frac{1}{2}}(\partial \Omega)$ to $H^{-\frac{3}{2}}(\partial \Omega)$ (see [BU02]). Using the a priori information of $\gamma_{j}$ again, (4.2) can be written as

$$
\left\|\partial_{\nu} \gamma_{1}-\partial_{\nu} \gamma_{2}\right\|_{L^{\infty}(\partial \Omega)} \leqslant C\left\|\tilde{\Lambda}_{\gamma}-\tilde{\Lambda}_{\gamma_{2}}\right\|_{*}^{\theta}
$$
that

With $q_{j}=\frac{\Delta \sqrt{\gamma_{j}}}{\sqrt{\gamma_{j}}}, j=1,2$, we want to estimate $\left\|\tilde{\Lambda}_{q_{1}}-\tilde{\Lambda}_{q_{2}}\right\|_{*}$ by $\left\|\tilde{\Lambda}_{\gamma_{1}}-\tilde{\Lambda}_{\gamma_{2}}\right\|_{*}$. Recall $\tilde{\Lambda}_{q}(f)=\left.\gamma^{-1 / 2}\right|_{\partial \Omega_{-, \varepsilon}}\left(\tilde{\Lambda}_{\gamma}\left(\left.\gamma^{-1 / 2}\right|_{\partial \Omega} f\right)+\left.\left.\partial_{\nu} \gamma^{\frac{1}{2}}\right|_{\partial \Omega_{-, \varepsilon}} f\right|_{\partial \Omega_{-, \varepsilon}}\right) \quad \forall f \in H^{\frac{3}{2}}(\partial \Omega)$.

We observe that $s+\frac{1}{2}>\frac{n-1}{2}+1$ and $s+\frac{3}{2}>\frac{n-1}{2}+2$. So by Sobolev's embedding theorem, the trace theorem, and (1.3), we get

$$
\begin{aligned}
& \left\|\gamma_{j}\right\|_{C^{1}(\partial \Omega)} \leqslant C_{1}\left\|\gamma_{j}\right\|_{H^{s+\frac{1}{2}}} \leqslant C_{1} N \\
& \left\|\gamma_{j}\right\|_{C^{2}(\partial \Omega)} \leqslant C_{2}\left\|\gamma_{j}\right\|_{H^{s+\frac{3}{2}}} \leqslant C_{2} N \\
& \left\|\partial_{\nu} \gamma_{j}\right\|_{C^{1}(\partial \Omega)} \leqslant C_{3}\left\|\partial_{\nu} \gamma_{j}\right\|_{H^{s+\frac{1}{2}}} \leqslant C_{3} N
\end{aligned}
$$

for $j=1,2$ and positive constants $C_{1}, C_{2}, C_{3}$. Thus we have that

$$
\begin{aligned}
\left\|\left(\tilde{\Lambda}_{q_{1}}-\tilde{\Lambda}_{q_{2}}\right) f\right\|_{H^{\frac{1}{2}}} \leqslant & C\left\|\gamma_{1}^{-\frac{1}{2}}-\gamma_{2}^{-\frac{1}{2}}\right\|_{C^{1}(\partial \Omega)}\left\|\tilde{\Lambda}_{\gamma_{1}}\left(\gamma_{1}^{-\frac{1}{2}} f\right)+\left.\left.\partial_{\nu} \gamma_{1}^{\frac{1}{2}}\right|_{\partial \Omega_{-, \varepsilon}} f\right|_{\partial \Omega_{-, \varepsilon}}\right\|_{H^{\frac{1}{2}}} \\
& +C\left\|\gamma_{2}^{-\frac{1}{2}}\right\|_{C^{1}(\partial \Omega)}\left(\left\|\tilde{\Lambda}_{\gamma_{1}}\left(\gamma_{1}^{-\frac{1}{2}} f\right)-\tilde{\Lambda}_{\gamma_{2}}\left(\gamma_{2}^{-\frac{1}{2}} f\right)\right\|_{H^{\frac{1}{2}}}\right. \\
& \left.+\left\|\partial_{\nu} \gamma_{1}^{\frac{1}{2}}-\partial_{\nu} \gamma_{2}^{\frac{1}{2}}\right\|_{C^{1}(\partial \Omega)}\|f\|_{H^{\frac{3}{2}}}\right) \\
\leqslant & C\left\|\gamma_{1}^{-\frac{1}{2}}-\gamma_{2}^{-\frac{1}{2}}\right\|_{C^{1}(\partial \Omega)}\left(\left\|\gamma_{1}^{-\frac{1}{2}}\right\|_{C^{2}(\partial \Omega)}+\left\|\partial_{\nu} \gamma_{1}^{\frac{1}{2}}\right\|_{C^{1}(\partial \Omega)}\right)\|f\|_{H^{\frac{3}{2}}} \\
& +C\left(\left\|\tilde{\Lambda}_{\gamma_{1}}\left(\gamma_{1}^{-\frac{1}{2}} f\right)-\tilde{\Lambda}_{\gamma_{2}}\left(\gamma_{2}^{-\frac{1}{2}} f\right)\right\|_{H^{\frac{1}{2}}}+\left\|\partial_{\nu} \gamma_{1}^{\frac{1}{2}}-\partial_{\nu} \gamma_{2}^{\frac{1}{2}}\right\|_{C^{1}(\partial \Omega)}\|f\|_{H^{\frac{3}{2}}}\right) \\
\leqslant & C\left(\left\|\gamma_{1}-\gamma_{2}\right\|_{C^{1}(\partial \Omega)}+\left\|\partial_{\nu} \gamma_{1}-\partial_{\nu} \gamma_{2}\right\|_{C^{1}(\partial \Omega)}\right)\|f\|_{H^{\frac{3}{2}}} \\
& +C\left\|\tilde{\Lambda}_{\gamma_{1}}\left(\gamma_{1}^{-\frac{1}{2}} f\right)-\tilde{\Lambda}_{\gamma_{2}}\left(\gamma_{2}^{-\frac{1}{2}} f\right)\right\|_{H^{\frac{1}{2}}} .
\end{aligned}
$$

We now estimate

$$
\begin{aligned}
\left\|\tilde{\Lambda}_{\gamma_{1}}\left(\gamma_{1}^{-\frac{1}{2}} f\right)-\tilde{\Lambda}_{\gamma_{2}}\left(\gamma_{2}^{-\frac{1}{2}} f\right)\right\|_{H^{\frac{1}{2}}} & \leqslant\left\|\tilde{\Lambda}_{\gamma_{1}}\left(\gamma_{1}^{-\frac{1}{2}}-\gamma_{2}^{-\frac{1}{2}}\right) f\right\|_{H^{\frac{1}{2}}}+\left\|\left(\tilde{\Lambda}_{\gamma_{1}}-\tilde{\Lambda}_{\gamma_{2}}\right)\left(\gamma_{2}^{-\frac{1}{2}} f\right)\right\|_{H^{\frac{1}{2}}} \\
& \leqslant C\left(\left\|\gamma_{1}-\gamma_{2}\right\|_{C^{2}(\partial \Omega)}+\left\|\tilde{\Lambda}_{\gamma_{1}}-\tilde{\Lambda}_{\gamma_{2}}\right\|_{*}\right)\|f\|_{H^{\frac{3}{2}}} .
\end{aligned}
$$

Next using Sobolev's embedding theorem together with the logarithmic convexity of Sobolev norms and the trace theorem, we obtain that

$$
\begin{aligned}
\left\|\gamma_{1}-\gamma_{2}\right\|_{C^{2}(\partial \Omega)} & \leqslant C\left\|\gamma_{1}-\gamma_{2}\right\|_{H^{s+\frac{3}{2}}(\partial \Omega)} \\
& \leqslant C\left\|\gamma_{1}-\gamma_{2}\right\|_{L^{2}(\partial \Omega)}^{\frac{2}{2+5}}\left\|\gamma_{1}-\gamma_{2}\right\|_{H^{s+\frac{5}{2}}(\partial \Omega)}^{\frac{2 s+3}{2 s+5}} \\
& \leqslant C\left\|\gamma_{1}-\gamma_{2}\right\|_{L^{\infty}(\partial \Omega)}^{\frac{2}{2+5}}\left\|\gamma_{1}-\gamma_{2}\right\|_{H^{s+5}(\Omega)}^{\frac{2 s+3}{2+5}} \\
& \leqslant C\left\|\gamma_{1}-\gamma_{2}\right\|_{L^{\infty}(\partial \Omega)}^{\frac{2}{2+5}} .
\end{aligned}
$$


In the same manner, we can get that

$$
\left\|\gamma_{1}-\gamma_{2}\right\|_{C^{1}(\partial \Omega)} \leqslant C\left\|\gamma_{1}-\gamma_{2}\right\|_{L^{\infty}(\partial \Omega)}^{\frac{2}{2 s+3}}
$$

and

$$
\left\|\partial_{\nu} \gamma_{1}-\partial_{\nu} \gamma_{2}\right\|_{C^{1}(\partial \Omega)} \leqslant C\left\|\partial_{\nu} \gamma_{1}-\partial_{\nu} \gamma_{2}\right\|_{L^{\infty}(\partial \Omega)}^{\frac{2}{2 s+3}} .
$$

Putting (4.1), (4.3)-(4.8) together yields

$$
\left\|\tilde{\Lambda}_{q_{1}}-\tilde{\Lambda}_{q_{2}}\right\|_{*} \leqslant C\left\|\tilde{\Lambda}_{\gamma_{1}}-\tilde{\Lambda}_{\gamma_{2}}\right\|_{*}^{\sigma}
$$

for some $0<\sigma<1$ with $\sigma=\sigma(n, s)$.

Now we want to estimate $\gamma_{1}-\gamma_{2}$ in $\Omega$. We get from [A188 (26) on p 168] that

$$
\left\|\gamma_{1}-\gamma_{2}\right\|_{L^{\infty}(\Omega)} \leqslant C\left(\left\|\gamma_{1}-\gamma_{2}\right\|_{L^{\infty}(\partial \Omega)}+\left\|q_{1}-q_{2}\right\|_{L^{\infty}(\Omega)}^{\tilde{\sigma}}\right)
$$

for some $0<\tilde{\sigma}<1$, where $C=C(N, \Omega)$ and $\tilde{\sigma}=\tilde{\sigma}(n, s)$. Hence, combining (4.1) and (1.2) gives

$$
\left\|\gamma_{1}-\gamma_{2}\right\|_{L^{\infty}(\Omega)} \leqslant C\left(\left\|\tilde{\Lambda}_{\gamma_{1}}-\tilde{\Lambda}_{\gamma_{2}}\right\|_{*}^{\frac{1}{2}}+\left\|\tilde{\Lambda}_{q_{1}}-\tilde{\Lambda}_{q_{2}}\right\|_{*}^{\sigma_{1} \tilde{\sigma}}+\left(\frac{1}{K} \log \left|\log \left\|\tilde{\Lambda}_{q_{1}}-\tilde{\Lambda}_{q_{2}}\right\|_{*}\right|\right)^{-\sigma_{2} \tilde{\sigma}}\right) .
$$

Finally, substituting (4.9) into (4.10) we arrive at

$$
\left\|\gamma_{1}-\gamma_{2}\right\|_{L^{\infty}(\Omega)} \leqslant C\left(\left\|\tilde{\Lambda}_{\gamma_{1}}-\tilde{\Lambda}_{\gamma_{2}}\right\|_{*}^{\tilde{\sigma}_{1}}+\left(\frac{1}{K} \log \left(\frac{1}{\sigma}\left|\log \left\|\tilde{\Lambda}_{\gamma_{1}}-\tilde{\Lambda}_{\gamma_{2}}\right\|_{*}\right|\right)\right)^{-\tilde{\sigma}_{2}}\right)
$$

for some $\tilde{\sigma}_{1}, \tilde{\sigma}_{2} \in(0,1)$. The proof of corollary 1.2 is now complete.

\section{Acknowledgments}

Horst Heck was financially supported by the Deutsche Forschungsgemeinschaft DFG. Jenn-Nan Wang was supported in part by the National Science Council of Taiwan (NSC 94-2115-M-002-003). This work was done when both authors were visiting the University of Washington. We would like to thank Gunther Uhlmann for his encouragements and the Department of Mathematics at the University of Washington for its hospitality. Also, we want to thank Mourad Bellassoued for calling our attention to an error in the early version of the paper.

\section{References}

[A188] Alessandrini G 1988 Stable determination of conductivity by boundary measurements Appl. Anal. 27 $153-72$

[A189] Alessandrini G 1991 Determining conductivity by boundary measurements, the stability issue Applied and Industrial Mathematics (Venice, 1989) (Math. Appl. vol 56) (Dordrecht: Kluwer) pp 317-24

[BU02] Bukhgeim A L and Uhlmann G 2002 Recovering a potential from partial Cauchy data Commun. Partial Diff. Equns 27 653-68

[Ca80] Calderón A 1980 On an inverse boundary value problem Seminar on Numerical Analysis and its Applications to Continuum Physics (Río de Janeiro: Soc. Brasileira de Matemática) pp 65-73

[GU01] Greenleaf A and Uhlmann G 2001 Local uniqueness for the Dirichlet-to-neumann map via the two-plane transform Duke Math. J. 108 599-617

[KSU05] Kenig C E, Sjöstrand J and Uhlmann G The Calderón problem with partial data Ann. Math. at press

[KV84] Kohn R V and Vogelius M 1984 Determining conductivity by boundary measurements Commun. Pure Appl. Math. 37 289-98

[Na88] Nachman A 1988 Reconstructions from boundary measurements Ann. Math. 128 531-76 
[Ma01] Mandache N 2001 Exponential instability in an inverse problem for the Schrödinger equation Inverse Problems 17 1435-44

[SU87] Sylvester J and Uhlmann G 1987 A global uniqueness theorem for an inverse boundary value problem Ann. Math. 125 153-69

[SU88] Sylvester J and Uhlmann G 1988 Inverse boundary value problems at the boundary-continuous dependence Commun. Pure Appl. Math. 41 197-219

[Tr95] Triebel H 1995 Interpolation Theory, Function Spaces, Differential Operators 2nd edn (Leipzig: Johann Ambrosius Barth, Heidelberg)

[Ve99] Vessella S 1999 A continuous dependence result in the analytic continuation problem Forum Math. 11 695-703 